"C 2019 IEEE. Personal use of this material is permitted. Permission from IEEE must be obtained for all other uses, in any current or future media, including reprinting/republishing this material for advertising or promotional purposes, creating new collective works, for resale or redistribution to servers or lists, or reuse of any copyrighted component of this work in other works." 


\title{
Multi-agent optimization approach to supply network configuration problems with varied product-market profiles
}

\author{
Subodha Dharmapriya ${ }^{*}$, Senevi Kiridena ${ }^{1}$, Nagesh Shukla ${ }^{2}$ \\ ${ }^{1}$ School of Mechanical, Materials, Mechatronic and Biomedical Engineering, Faculty of Engineering and \\ Information Sciences, University of Wollongong, NSW 2522, Australia \\ ${ }^{2}$ School of Information, Systems and Modelling, Faculty of Engineering and Information Technology, \\ University of Technology Sydney, NSW 2007, Australia \\ *Corresponding Author: ssd889@,uowmail.edu.au
}

\begin{abstract}
This paper demonstrates the application of a novel multi-agent modelling
\end{abstract} approach to support supply network configuration (SNC) decisions towards addressing several challenges reported in the literature. These challenges include: enhancing supply network (SN)-level performance in alignment with the goals of individual SN entities; addressing the issue of limited information sharing between SN entities; and sustaining competitiveness of SNs in dynamic business environments. To this end, a multi-stage, multi-echelon SN consisting of geographically dispersed SN entities catering to distinct product-market profiles was modelled. In modelling the SNC decision problem, two types of agents, each having distinct attributes and functions, were used. The modelling approach incorporated a reverse-auctioning process to simulate the behaviour of SN entities with differing individual goals collectively contributing to enhance SN-level performance, by means of setting reserve values generated through the application of a genetic algorithm. A set of Pareto-optimal SNCs catering to distinct product-market profiles was generated using Non-dominated Sorting Genetic Algorithm II. Further evaluation of these SNCs against additional criteria, using a rule-based approach, allowed the selection of the most appropriate SNC to meet a broader set of conditions. The model was tested using a refrigerator $\mathrm{SN}$ case study drawn from the literature. The results reveal that a number of SNC decisions can be supported by the proposed model, in particular, identifying and evaluating robust SNs to suit varied product-market profiles, enhancing SC capabilities to withstand disruptions and developing contingencies to recover from disruptions. 
Keywords - supply network dynamics, supply chain design, supply network configuration

Managerial relevance: Compared to the existing SNC decision support tools, the proposed modelling approach addresses three key challenges faced by decision makers. First, it ensures optimal SN-level performance when individual SN entities still aiming to satisfy their local goals such as organisation-specific competitive priorities. Second, it facilitates SNC decisionmaking leading to optimal SN-level performance with minimal information sharing among SN entities, which reflects the real-world situation of organisations' reluctance to disclose commercially sensitive information. Third, the model can be useful in facilitating SNC decisions to sustain competitiveness of SNs in a dynamic business environment, which is characterised by changing consumer requirements, disruptions and other forms of uncertainty. Overall, the proposed multi-agent optimisation model can be used to enhance SNC decisions by any $\mathrm{SN}$ entity, as well as other parties such as supply chain analysts or consultants.

\section{INTRODUCTION}

Facilitated by ongoing advances in technology and information systems, as well as the pursuit of broad-based initiatives such as Industry 4.0, production networks are becoming more distributed and globally dispersed [1-2]. Additionally, shifting product-market profiles and evolving competitive dynamics demand ongoing adjustments to supply chain (SC) structures [3]. The combined effects of these developments mean that appropriately responding to both unforeseen and anticipated disruptions is critical to maintaining fast, efficient and responsive SCs. The capacity for timely responding to these disruptions can be built through measures aimed at improving SC resilience and robustness as part of design considerations or operational control. Our review of the literature on SC disruptions indicates that, compared to the work undertaken in the area of unforeseen disruptions, efforts directed towards the development of comprehensive responses to anticipated disruptions are sparse. 
Therefore, in this study, we propose a multi-agent optimization approach to support supply network configuration (SNC) decisions involving varied product-market profiles. In so doing, we illustrate how such an approach could contribute to model the individual behaviour of supply network (SN) entities, as well as the impact of their decisions on the overall SN-level performance. The paper is organized as follows. Following this brief introduction, we present a summary review of the extant literature on the SNC problem, while also focusing on measures aimed at addressing SC disruptions. Next, the conceptual framework that guided the development of the proposed approach to solving the SNC problem is presented. This is followed by a detailed account of the proposed multi-agent optimization model (MAOM), including the way it is used in solving the SNC problem. We then test the proposed MAOM using a case study of a refrigerator production network drawn from the literature.

\section{LITERATURE REVIEW}

SC disruptions reported in the literature include unforeseen incidents such as transportation mishaps, natural calamities, and intentional attacks [4], as well as anticipated circumstances such as facility breakdowns, failures of the supplier base, offensive actions of competitors and major shifts in demand [5-6]. Some authors have classified such events in terms of endogenous disruptions and exogenous disruptions [7]. Irrespective of the way they are classified, all disruptions are known to induce significant risks in terms of their impact on SC functioning or performance. Mitigating the impact of such risks involves improving SC robustness and resilience through building capacity to: withstand disruptions, including any structural adjustments required; respond quickly to disruptions; and recover effectively from any disruptive incidents [8-9]. Specific measures of risk mitigation that have been proposed in the literature include: strategies such as holding buffer stocks, maintaining back-up capacity, multiple-sourcing and adaptive ordering [4-5]; structural adjustments in SNs [3]; and dynamic integration of logistics capabilities [10]. For instance, Hasani and Khosrojerdi [11] proposed 
six measures covering both operational strategies and structural adjustments to deal with SC disruptions and uncertainty, namely, facility dispersion, facility reinforcement, use of subassemblies, multiple sourcing, keeping inventory and considering primary and alternative bills of material (BOM). Overall, the literature cited above highlights the existence of an array of measures that could potentially be used to mitigate the risks associated with SC disruptions, through building SC robustness and resilience. Furthermore, it is advocated that such capacity must be built through SNC decisions leading to targeted proactive and reactive actions.

In general, the term SC refers to the sequential arrangement of organizational entities involved in acquiring raw materials, transforming them into components and assemblies, and then distributing the final product to end-users. However, given that an organizational entity can be part of more than one $\mathrm{SC}$, a more realistic alternative would be to consider the notion of SN. With the notion of SN comes the existence of multiple SCs that are capable of catering to a given product-market profile. This then introduces the possibility of differentiating such SCs based on their performance in terms of speed, efficiency and responsiveness. The common industry practice may be to use the same set of SN entities catering to a given product-market profile for a prolonged period, considering such factors as the benefits of maintaining longterm relationships, contractual arrangements and ease of coordination and communication [12]. On the one hand, adhering to the same SC for too long can lead to the loss of competitiveness at the SC level, because developments such as the emergence of high-performing SC entities, the adoption of new technology and the introduction of new and better substitute products can significantly alter the overall competitiveness of alternative SCs [13]. On the other hand, shifting product-market profiles means a SC that has been configured to serve a given productmarket profile at a particular point in time could become less competitive if it is no longer aligned with the current product-market profile [14-15]. Therefore, SNC decisions can play a 
critical role in sustaining the overall SC performance under evolving conditions associated with globally dispersed SNs, as well as in the face of disruptions $[13,16]$.

SNCs are alternative arrangements of organizational entities, processes and resources where, as a whole, they can be differentiated based on key performance metrics such as cost, lead-time and fill rate [17-18]. Previous studies have addressed SN design issues related to the decisions involving facility location, supplier selection and the optimal number of facilities required, often assuming static conditions [19-20]. However, more recent literature has contributed to accounting for disruptions and uncertainties in the design stage of SNs by introducing stochastic programming to the modelling environment [21]. Recent reviews of SNC literature [22-23] reveal that the spatial (e.g. geographical dispersion) and temporal (e.g. disruptions, market dynamics) aspects of SNs are particularly under-researched. For example, even though the product-market profile is an important factor to consider in SNC decisions, changes in product-market profile over time have not been adequately addressed in the literature. Similarly, although a particular market segment could be represented using multiple attributes such as demand, lead-time and willing-to-pay price, the treatment of product-market profile in most existing SN literature only accounts for the attribute of demand [3].

Consistent with the approaches used in related areas such as $\mathrm{SN}$ design and logistics network planning [24], optimization techniques have played a major role in SNC research. In most cases, SNC problems are treated as combinatorial optimization type aimed at finding optimal SNC(s) based on the desired performance attributes of SN entities. As such, researchers have often used meta-heuristics for solving SNC problems. Among these approaches, Genetic Algorithm [e.g.18], Ant Colony Optimization [e.g. 25] and Bee Algorithm [e.g. 26] are the most widely used [16]. The Ant Colony Optimization and Bee Algorithm-based studies have accounted for multiple objectives such as lead-time and cost. Multi-agent systems (MAS) have been proposed in the literature to deal with SNC problems requiring particular attention to 
coordination issues. These studies have used techniques such as argumentation-based negotiation [27], contract net protocol (CNP) with negotiation [28] and case-based reasoning, along with CNP [29]. Opportunities for using Real Options (RO), along with Game-theoretic approaches to model SN entity behaviour have also been pointed out more recently [13].

A number of studies [30-31] have addressed SNC decisions rather holistically in the context of multi-tiered SNs, but without considering the individual SN entity-level decisions. The majority of such studies [27-29; 32-33] have also been undertaken in the context of rather narrowly defined SNs with limited SC tiers (mostly upstream of the SC) and simple product structures. To model SNC problems in more realistic terms, integration of SN-entity (local) decision-making and SN-level (global) performance is required [34]. However, integration of these perspectives cannot be achieved by using either meta-heuristics, multi-agent systems or any other similar technique, alone, hence suggesting the need for a combined approach.

Overall, the literature points to a number of limitations in the current approaches to modelling structural, spatial and temporal characteristics of SNs, which could be summarised as: models with limited number of tiers, $\mathrm{SN}$ entities and product flows, including lack of recognition of geographical dispersion; and not accounting for multiple and dynamic operational parameters such as cost and lead-time, as well as changing product-market profiles. This study endeavours to address these limitations by focusing on multi-echelon SNs consisting of geographically dispersed $\mathrm{SN}$ entities that independently make decisions in responding to varied product-market profiles. It is expected that modelling SN structures and SN-entity level decision-making more realistically will help observe and study the performance implications of various SNC decisions, at the SN-level, in a meaningful and holistic manner.

\section{Conceptual framework}

The overall aim of this study is to develop a modelling approach to support SNC decisions while accounting for varied product-market profiles under a given set of organizational and 
environmental conditions. The overall conceptual framework used in the development of the proposed methodology is depicted in Figure 1, which consists of the three steps listed below.

(i) Establish the product-market profiles representing different consumer regions;

(ii) Generate alternative SNCs catering to specific product-market profiles; and

(iii) Evaluate the SNCs generated in step (ii) against a set of metrics representing the chosen competitive priorities or performance criteria applicable to a given context.

In relation to the above framework, the proposed modelling approach primarily concerns with the MAOM, which accounts for the aspects included in the shaded box in Figure 1. However, to help illustrate the application of the MAOM, the remaining aspects of the framework are also briefly introduced in this section.

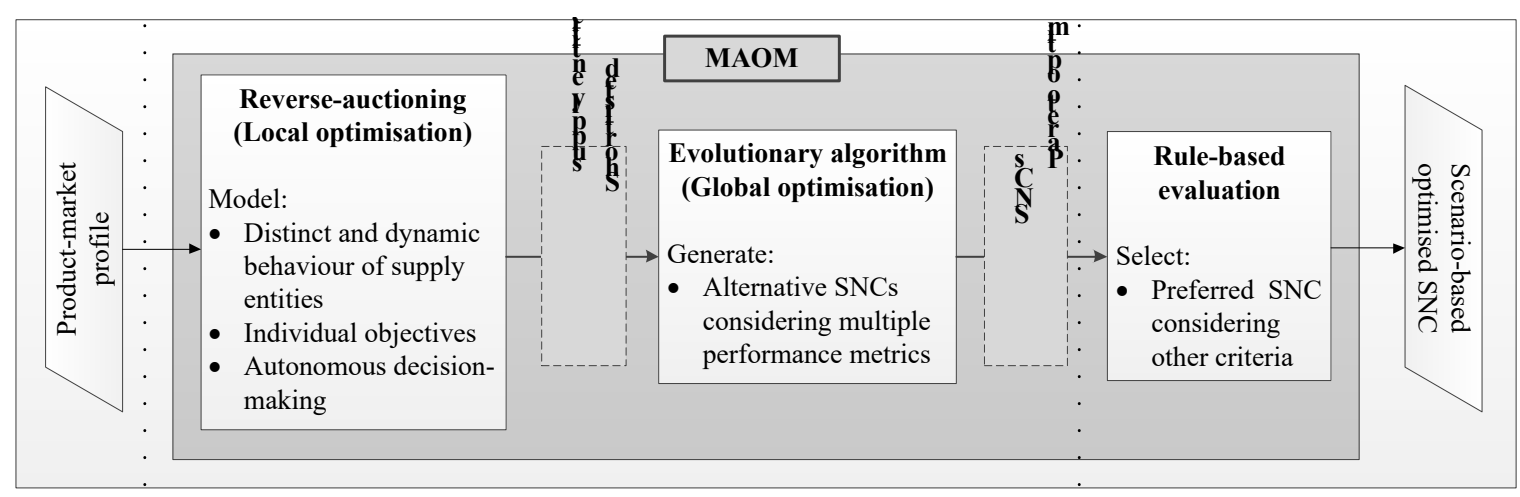

Figure 1: Conceptual framework guiding the proposed methodological approach

\subsection{ESTABLISING PRODUCT-MARKET PROFILES}

The product-market profile of a given consumer region $l$ represents the estimated consumer requirements with respect to three key attributes, aggregate demand $\left(V_{l}\right)$, expected lead-time $\left(L T_{l}\right)$ and willing-to-pay price $\left(P_{l}\right)$. The common practice is to derive the values of such attributes using demographic and historical data. Given that such historical data is not readily available to be used in this study, we use a set of alternative methods, as briefly outlined below.

The base parameters used to determine the values of the three attributes referred to above are per-capita income [35], price level index (i.e. the ratio of purchasing power parity to market exchange rate) [36], energy consumption [37] and population density [38]. Analytical 
Hierarchy Process (AHP), a widely used multi-criteria problem solving technique, is used to estimate $V_{l}$, using all four base parameters. A more detailed account of the approach followed in determining the values of $V_{l}$ is provided in Appendix I. The use of AHP for estimating $V_{l}$ allows accounting for multiple dimensions that characterise a given consumer region, as well as the key differences between consumer regions, to arrive at a more robust estimate of the aggregate demand, compared to assuming that the demand is proportional to the population density of the target consumer region alone. Attribute $P_{l}$ for the target consumer region is taken as proportionate to the price level index of that region. Attribute $L T_{l}$ is estimated based on percapita income, assuming that populations with high income (i.e. affluent consumers) expect a shorter delivery lead-time; higher the income, shorter the expected lead-time.

\subsection{GENERATION OF ALTERNAITVE OPTIMAL SNCS}

SNs consist of a number of autonomous or semi-autonomous business organizations (i.e. SN entities) with distinct characteristics such as capabilities, resources and processes, which function based on a set of competitive priorities. Typically, these SN entities independently make decisions while interacting with other $\mathrm{SN}$ entities and take necessary actions (e.g. adopting new technologies, expanding the capacity of facilities and updating business models) to cope with the challenges such as market forces or competitor manoeuvres [39]. Such distinct and dynamic behaviour of individual SN entities can create complex aggregate behaviour at the SN level. It is the cumulative effect of these collective decisions and actions that manifests in the form of SNs that are competing against each other in terms of satisfying a given productmarket profile. As such, to arrive at an optimal solution for a given SNC problem, both the SN-entity (local) decision-making and SN-level (global) performance need to be aligned. The challenging nature of such problems (e.g. distributed decision-making in a global context) demands solution approaches that extend beyond the realm of mathematical programming [40]. 
Therefore, this study proposes a multi-agent modelling approach consisting of reverseauctioning and evolutionary algorithms with the aim of determining the optimal SNCs catering to a specific product-market profile, while accounting for the diverse goals and autonomous decision-making behaviour of individual SN entities of a given SN. The reverse-auctioning process serves two purposes in relation to identifying a set of $\mathrm{SN}$ entities: one is to determine reserved values for each node in the bidding process with the aim of optimizing SN-level performance; and the other is to ensure that a competitive bidding process takes place.

\subsection{SCENARIO-BASED EVALUATION OF SNCS}

Pertaining dynamics (e.g. uncertainties in operational parameters, disruptions and structural changes of SN) and inter-dependencies between various SN entities make SN design decisions such as supplier selection, facility location and order allocation particularly challenging. Among the mix of modelling methods and solution methodologies proposed in the literature (e.g. stochastic modelling, simulation and game theory), evaluating "what-if" situations supported by scenario-based approaches are considered to be appropriate for solving SNC problems under dynamic conditions [22]. The proposed approach first determines alternative optimal SNCs considering a selected set of SN-level performance metrics for a given productmarket profile and these optimal SNCs are then evaluated against additional criteria (e.g. supply reliability, supplier compatibility and sustainability). We consider efficiency and speed as two key SN-level performance metrics against which the alternative optimal SNCs are evaluated initially. These SNCs may then be subject to further evaluation based on other criteria such as technology integration, energy consumption or carbon footprint, as needed.

\section{PROPOSED MULTI-AGENT MODELLING APPROACH}

Two major views on 'what an agent is' can be gleaned from the extant literature: one focusing on the attributes and behaviours of agents and the other focusing on their applications; 
i.e. how agents are used in solving problems within a particular domain [41-43]. Based on our review of the definitions currently available in the literature, we consider agents to be entities representing human representatives or tasks (mutually exclusive) with certain inherent characteristics, which are typically executed using software applications. The literature has reported successful implementations of multi-agent modelling approaches for a variety of applications in different disciplines [40,44]. Such applications are identified in the literature as modular, decentralized, changeable, ill-structured and complex [45]. The salient features of the modelling approaches used in these applications can be differentiated in terms of the agent environment, agent attributes, agent characteristics and agent architecture. We follow a similar approach in developing the proposed MAOM, as further elaborated below.

\subsection{AGENT ENVIRONMENT}

Agent environment is defined in the literature as the modelling context that falls outside the control of agents [46-47]. In relation to the SNC problem, we consider individual SN entities as agents and the SN environment as the agent environment. The $\mathrm{SN}$ environment can be explained in terms of structural and spatial characteristics. The structural dimension of the environment reflects the composition of the SN, which includes: the number of tiers in the SN; the number of SN entities in different tiers and their relationships; and multiple product platforms and product variants involved [48]. The spatial dimension of the environment represents the geographical dispersion of SN entities for a given SN. These aspects are reflected in the way SN entities are arranged into a typical SN illustrated in Figure 2.

The typical SN considered in this study has $I$ number of stages $(\mathbf{S})$ where $\mathbf{S}=\left(\mathbf{S}_{1} \ldots \mathbf{S}_{i \ldots} \ldots \mathbf{S}_{I}\right)$ and $\mathbf{S}_{i}$ is the $i^{\text {th }}$ stage of the $\mathrm{SN}$, covering the entire value-adding chain. As such, a given stage $\mathbf{S}_{i}$ could be responsible for: either supplying raw materials; producing parts, components or sub-assemblies; assembling final products; or delivering finished goods through various intermediate points to the final consumer. There can be multiple nodes at any given stage (for 


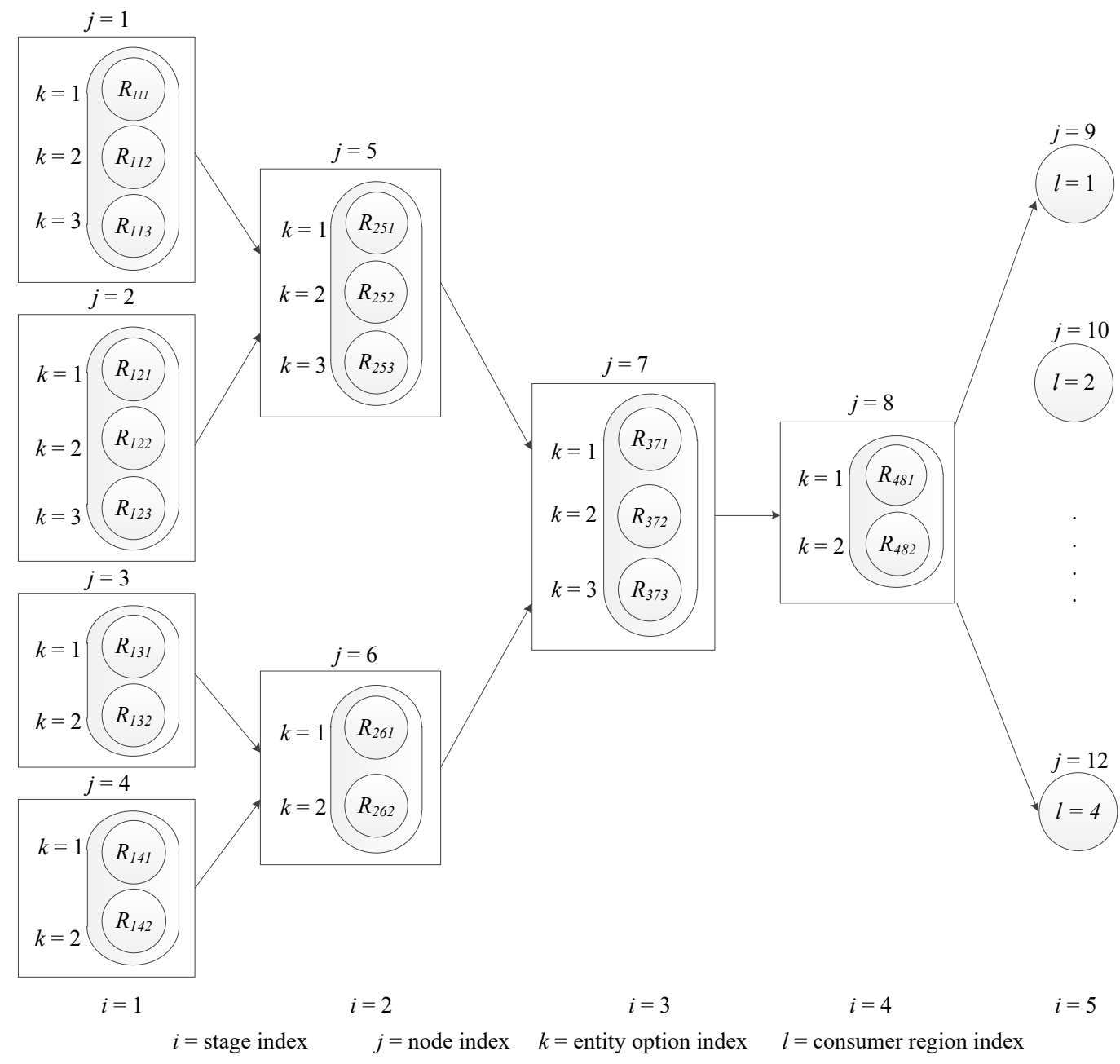

Figure 2: Representation of SN environment

example, multiple types of components or raw materials sourced at the initial stage). We assume that there are $J$ nodes, in total, in the SN. Therefore, $j^{\text {th }}$ node at the $i^{\text {th }}$ stage of the SN can be represented by $\mathbf{N}_{i j}$. Furthermore, at a given node $\mathbf{N}_{i j}$, there can be several competing SN entities (i.e. entity options), represented by $\boldsymbol{R}_{i j k}$ (where $k \in\left(1,2,3 \ldots p_{j}\right)$ ), which perform the same function (e.g. supplying a particular type of component or the same raw material). Here, $p_{j}$ is the maximum number of entity options available at the $j^{\text {th }}$ node.

These entity options are capable of performing the required value-adding functions at the respective node. Depending on factors such as the location of facilities, the capacity of plants and the processes or technologies utilized, these entity options can compete with each other on the basis of cost, lead-time and quality etc. For example, a local entity option may be able to 
supply a component at a higher price with a shorter lead-time, whereas an overseas supplier may be able to supply it at a much lower price but with a considerably longer lead-time. Additionally, consumers are located in different geographical regions and we assume that there are $L$ number of consumer regions, for the problem considered in this study.

\subsection{AGENT CHARACTERISTICS AND ARCHITECTURE}

In line with the way agents are defined, a range of agent characteristics are presented in the literature. In this study, we adopted the suite of characteristics proposed by Wooldridge and Jennings [49] and interpret each of them here with respect to the SN context introduced above.

- Autonomous: SN entities make their own decisions considering their competitive priorities, organisational strategies and available resources.

- Adaptive: SN entities change their behaviour/decisions in light of external factors such as business trends, regulatory frameworks and economic conditions.

- Reactive: SN entities timely respond to the external influences such as plant breakdowns, loss of suppliers and transport delays with appropriate actions.

- Pro-active: SN entities review their operations from time to time and implement new initiatives (e.g. capacity additions) to cope up with future changes.

- Social: SN entities interact with each other for fulfilling customer requirements, for example, in relation to placing orders for raw materials and parts.

Agent architecture is another important aspect of the modelling approaches used, which reflects how an agent is constructed thus giving rise to certain properties, as well as behavioural or functional attributes [50-51]. Maes [51] proposed a succinct definition of agent architecture, a collection of modules with a mechanism to interact with each other to perform a particular function, which we chose to adopt. In this study, agents consist of multiple modules such as decisions-making module (DM), learning module (LM) and communication module (CM). 


\subsection{AGENT TYPES AND BEHAVIOUR}

In the literature, agent classifications are proposed based on the role of the agent within the system concerned [52]. In this study, we introduced two types of agents namely, physical and auxiliary. We consider SN entities (e.g. suppliers, manufactures) performing typical SN operations as physical agents and those who support SNC decision-making as auxiliary agents.

\subsubsection{PHYSICAL AGENTS}

In relation to the SNC problem studied, we introduce a set of three physical agents, namely, supplier agent (SA), manufacturer agent (MA) and distributor agent (DA) to represent suppliers, manufacturers and distributors of the SN respectively. These physical agents may be located in different geographical regions, and they are identified with an index indicating their stage $(i)$, node $(j)$ and entity option $(k)$ within the SN concerned respectively. The primary function of these agents is to perform the core value-adding operations in catering to a given product-market profile. As such, SAs are arranged into a number of tiers according to the BOM of the product involved. For example, if there are multiple tiers in the supply stage; the firsttier suppliers supply sub-assemblies; the second-tier suppliers supply the required parts and/or components; and the third-tier suppliers supply raw materials. Similarly, MAs assemble final products and DAs are responsible for storing, sorting and dispatching finished products to relevant consumer regions. These physical agents have distinct capacity levels $\left(A C_{i j k}\right)$ applicable to their value-adding operations (e.g. processing, assembly, storage and handling) depending on the SN node they belong to. Additionally, certain physical agents periodically increase their capacity by an increment expressed as a percentage of the current capacity, through purchase of new machineries, adopting new technology and expanding facilities etc. Given such capacity levels, each agent will then have a distinct unit operations $\operatorname{cost}\left(P C_{i j k}\right)$ and unit operations time $\left(P T_{i j k}\right)$ related to processing, assembly, storage and handling. 
In addition to performing core value-adding functions, these physical agents take part in the reverse-auctioning process to explore business opportunities. The functionality of physical

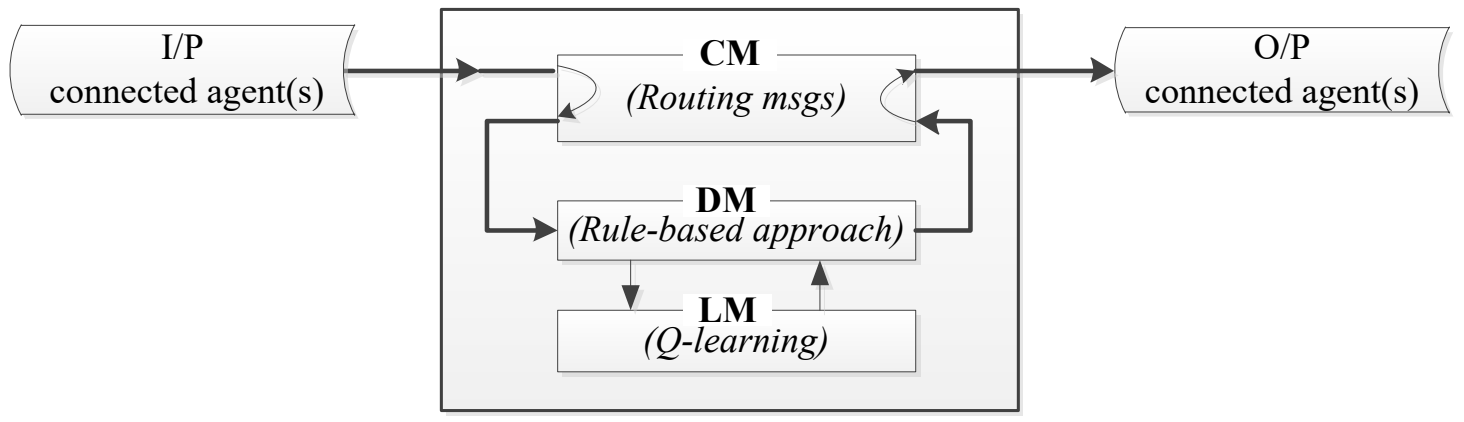

Figure 3: The architecture of physical agents

agents in the context of reverse-auctioning is executed with the help of the three modules DM, LM and CM, as shown in Figure 3.

Physical agents bid in terms of unit price $\left(B P_{i j k}\right)$ and unit time $\left(B T_{i j k}\right)$ corresponding to a given product-market profile, upon receiving an invitation to submit bids. When bidding, physical agents consider the state of their available capacity and past bidding outcomes. The overall bidding process, which may involve a pre-determined number of iterations, as illustrated in Figure 4, is modelled in the form of sequential decision-making following the Markov Decision Process (MDP), where Reinforcement Learning (RL)-based techniques are used to solve the MDP in relation to handling different bidding outcomes [53-54].

Upon receiving an invitation to bid, the DM of physical agents first chooses to follow either an exploration or an exploitation strategy, depending on whether the invitation is for a new product-market profile or not (i.e. whether they have bid in the past). In both cases, the physical agent generates a bid (i.e. the values of $B P_{i j k}$ and $B T_{i j k}$ ) for the first round of bidding, considering the desired profit margin, cost, time and the current capacity status. The current capacity status $m$ is determined situationally following a rule-based reasoning approach using Eqns. (1) and (2), supported by the Q-table shown in Table 1.

$$
\begin{gathered}
A A C_{i j k}=\left(1+\lambda_{i j k^{-}}^{1} \lambda_{i j k}^{2}\right) \times A C_{i j k} \\
R C_{i j}=\delta_{i j} \times V_{l}
\end{gathered}
$$


Table 1: Illustration of the Q-table*

\begin{tabular}{|c|c|c|c|c|}
\hline \multicolumn{2}{|c|}{ Action/Profit range $(n)$} & $\begin{array}{c}\text { Low } \\
(5-10 \%)\end{array}$ & $\begin{array}{c}\text { Medium } \\
(10-15 \%)\end{array}$ & $\begin{array}{l}\text { High } \\
(15-20 \%)\end{array}$ \\
\hline \multirow{2}{*}{$\begin{array}{l}\text { Under-utilized } \\
A A C_{i j k} \geq 0.5 \times N C_{i j k}\end{array}$} & $A A C_{i j k} \geqslant R C_{i j}$ & $\mathrm{Q}_{11}(5 \%)$ & $\mathrm{Q}_{12}(10 \%)$ & $\mathrm{Q}_{13}(15 \%)$ \\
\hline & $A A C_{i j k}<R C_{i j}$ & $\mathrm{Q}_{21}(6 \%)$ & $\mathrm{Q}_{22}(11 \%)$ & $\mathrm{Q}_{23}(16 \%)$ \\
\hline \multirow{2}{*}{$\begin{array}{l}\text { Utilized } \\
\left(0.25 \times N C_{i j k}\right) \leq A A C_{i j k}<\left(0.5 \times N C_{i j k}\right)\end{array}$} & $A A C_{i j k} \geqslant R C_{i j}$ & $\mathrm{Q}_{31}(7 \%)$ & $\mathrm{Q}_{32}(12 \%)$ & $\mathrm{Q}_{33}(17 \%)$ \\
\hline & $A A C_{i j k}<R C_{i j}$ & $\mathrm{Q}_{41}(8 \%)$ & $\mathrm{Q}_{42}(13 \%)$ & $\mathrm{Q}_{43}(18 \%)$ \\
\hline \multirow{2}{*}{$\begin{array}{l}\text { Fully-utilized } \\
A A C_{i j k}<\left(0.25 \times N C_{i j k}\right)\end{array}$} & $A A C_{i j k} \geqslant R C_{i j}$ & $\mathrm{Q}_{51}(9 \%)$ & $\mathrm{Q}_{52}(14 \%)$ & $\mathrm{Q}_{53}(19 \%)$ \\
\hline & $A A C_{i j k}<R C_{i j}$ & $\mathrm{Q}_{61}(10 \%)$ & $\mathrm{Q}_{62}(15 \%)$ & $\mathrm{Q}_{63}(20 \%)$ \\
\hline
\end{tabular}

*each cell contains a Q-value $\left(\mathrm{Q}_{m n}\right)$ corresponding to capacity status $m$ and profit range $n$. Percentage values in each cell are read as profit margins $\left(P_{m n}\right)$ for capacity state $m$ and action/profit range $n . N C_{i j k}$ represents planned annual capacity

Equation (1) refers to the available annual capacity $A A C_{i j k}$ which is the difference between planned annual capacity (where, $\lambda_{i j k}^{1}$ is the size of capacity addition, expressed as a percentage of the current annual capacity) and utilised capacity expressed as a percentage $\left(\lambda_{i j k}^{2}\right)$ of current annual capacity. Equation (2) refers to the $R C_{i j}$, which is the number of units required from node $j$ (i.e. $\delta_{i j}$ ), according to the BOM and demand $\left(V_{l}\right)$, as per the product-market profile. Once the relevant capacity status $m$ is determined, the corresponding profit range $n$ is read from the Q-table leading to the preferred action $A_{n}^{t}$, depending on the iteration $t$ of the auctioning process, at which the bid is considered and the experience of the agent reflected in the Q-value.

Initially, at iteration 1 , action $A_{n}^{t}$ is taken following either the exploration or exploitation strategy, as per the conditions shown Eqn. (3).

$$
A_{n}^{t}=\left\{\begin{array}{cc}
\operatorname{random}\left(A_{1 \rightarrow n}^{t}\right), & \text { if exploration } \\
\max \left(Q_{m n}^{t}\right), & \text { if exploitation }
\end{array}\right.
$$

Exploration strategy is appropriate in the case of a new product-market profile due to the absence of prior bidding outcomes, in situations such as the introduction of a new product, a new physical agent joining the SN or an existing physical agent bidding for the first time. 
Exploitation strategy is employed to make use of the physical agent's experience acquired through participation in past auctions or the earlier iterations of the current reverse-auction process. Under exploitation strategy, once action $A_{n}^{t}$ is selected, $B P_{i j k}$ and $B T_{i j k}$ are calculated as per Eqns. (4) and (5). $B P_{i j k}$ is calculated taking $P C_{i j k}$ and relevant $P_{m n}$ with respect to the capacity status $(m)$ and profit range $(n) . B T_{i j k}$ is calculated considering $P T_{i j k}$ and a coeficient $\left(\beta_{m}\right)$. The coefficient $\beta_{m}$ is introduced to account for the variation in production time with respect to capacity status. To illustrate this variation, $\beta_{m}$ values representing 6 states reflecting the three utilization levels shown in Table 1 are used; i.e. $\beta_{m ; m=1 \rightarrow 6}=\{1,1.15,1.3,1.45$, $1.6,1.75\}$.

$$
\begin{gathered}
B P_{i j k}=P C_{i j k}\left(1+P_{m n}\right) \\
B T_{i j k}=\beta_{m} \times P T_{i j k}
\end{gathered}
$$




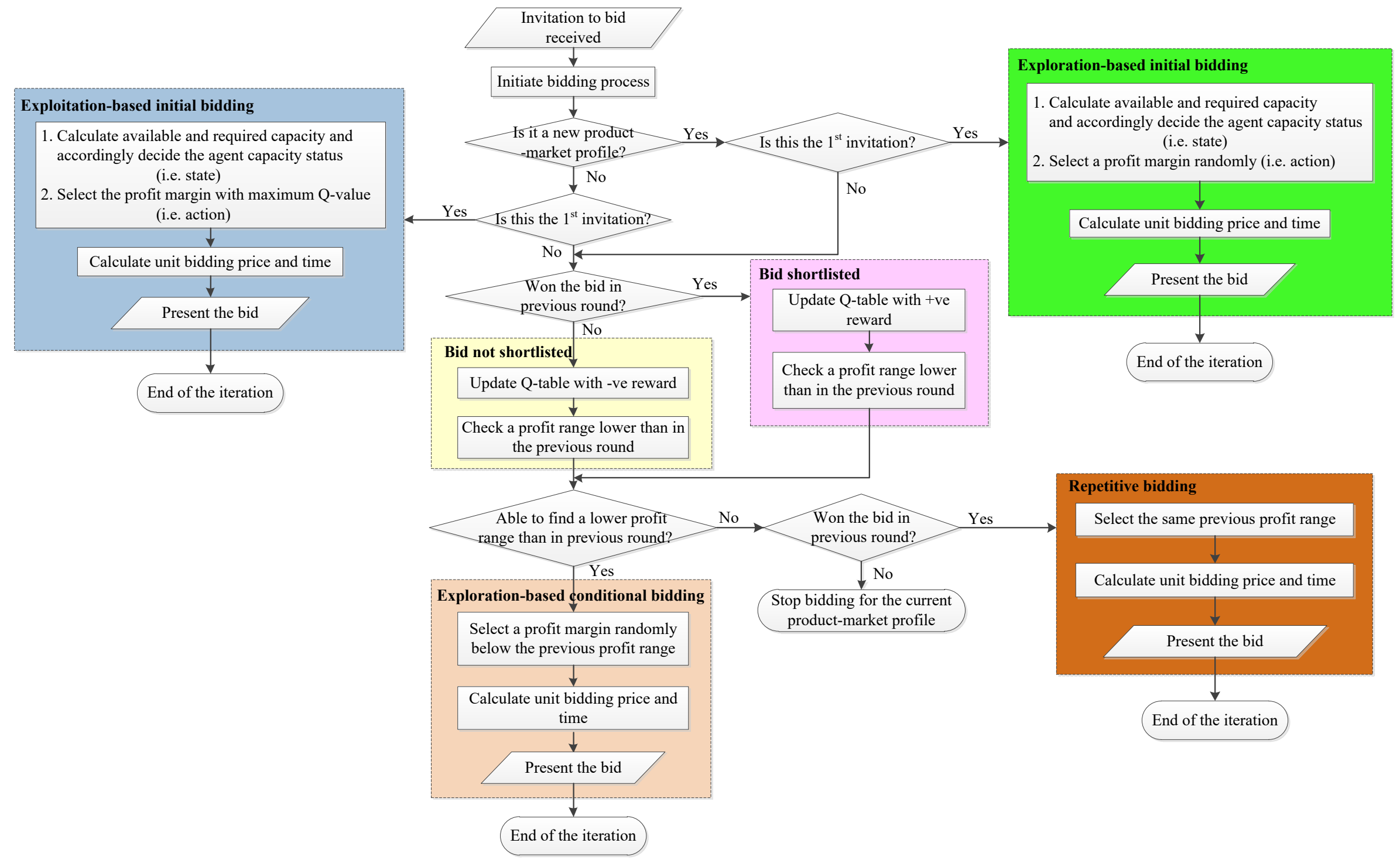

Figure 4: Steps involved in the decision-making process (bidding process) of physical agents 
Once the bids generated as above are presented to the corresponding SN entity selection agent (SES) for considearion, iteration 1 of the bidding process is complete. Upon evaluation of all bids received in iteration 1, the SES informs respective physical agents as to whether they are invited to bid in the next iteration of the auctioning process. Shortlisting of bids to proceed to the next iteration is made based on the comparison of bids received against the reserved values of price $\left(R P_{i j}\right)$ and time $\left(R T_{i j}\right)$ in the concluded iteration. Subsequent iterations of the bidding process may follow multiple paths as illustrated in Figure 4, depending on the outcomes of the previous iteration, as elaborated below.

At the start of each subsequent iteration, the physical agents update their Q-table based on the outcome in the previous iteration, as outlined below. Depending on whether or not the bid was shortlisted to proceed to the next iteration, the relevant Q-value, $Q_{m n}^{t}$, (i.e. corresponding to relevant state $m$ and action $n$ in iteration $t$ ) of the Q-table is updated with a positive or negative reward as per Eqns. (6) and (7).

$$
\begin{gathered}
Q_{m n}^{t}=Q_{m n}^{t-1}+\mu_{i j k}^{1}\left(\delta_{i j} \times V_{l} \times P_{m n}\right)+\gamma \times \max \left(Q_{m n}^{t+1}\right) \\
Q_{m n}^{t}=Q_{m n}^{t-1}-\mu_{i j k}^{2}\left(\delta_{i j} \times V_{l} \times P_{m n}\right)
\end{gathered}
$$

Here, $\mu_{1}$ is the percentage contribution of the overall profit corresponding to the previous action, $\gamma$ is the discount factor, which is applied to future rewards (represented as $\max \left(Q_{m n}^{t+1}\right)$ for iteration $t+1)$, where, $\mu_{2}$ is the percentage contribution of the overall loss $\left(\mu_{i j k}^{1} \geq \mu_{i j k}^{2}\right)$.

The physical agents then read the updated Q-table to see if there is a lower profit range available than that was used to bid in iteration $t$-1 (i.e. $n>1$ in iteration $t-1$ ). If a lower profit range in iteration $t-1$ can be found, then an exploration-based conditional bidding strategy is followed. Under this strategy, the physical agent randomly select an action, $A_{n}^{t}$, based on profit range $\left(A_{n}^{t-1}\right)$, which is less than that used in the previous iteration, as per Eqn. (8) and bidding values are again calculated according to Eqns. (4) and (5). 


$$
A_{n}^{t}=\operatorname{random}(A) \quad \mid A=\left(A_{1}^{t-1}, \ldots A_{n-1}^{t-1}\right) \quad \forall t>1
$$

In case that a lower profit range cannot be found, the physical agent considers whether the bid in the previous iteration was shortlisted or not. If the bid was shortlisted, then the repetitive bidding strategy is followed. Under this strategy, the physical agent presents the same values used in the previous iteration of auctioning in response to the current invitation, i.e.:

$$
A_{n}^{t}=A_{n}^{t-1} \quad \forall t>1
$$

Otherwise, the agent decides to stop further bidding for the given product-market profile, which brings the reverse-auctioning process to its conclusion.

\subsubsection{AUXILIARY AGENTS}

We introduce a set of six auxiliary agents, namely SES, order processing agent (OP), auctioning agent (AU), optimization agent (OPT), transportation agent (TA) and evaluation agent (EA). The role of auxiliary agents is to support SNC decisions in relation to the generation, optimization and evaluation of alternative SNCs for different product-market profiles. The way in which each of these agents functions is further elaborated below.

The OP processes product-market profile information to determine the number of units required at each supply node (i.e. $R C_{i j}$ ) taking into account both $V_{l}$ and $\delta_{i j}$ as per Eqn. 2. For iteration 1, the $\mathrm{AU}$ generates $R P_{i j}$ and $R T_{i j}$ for relevant $\mathrm{SN}$ nodes corresponding to a given product-market profile using the Genetic Algorithm (GA). The initial population for the GA is a set of $R P_{i j}$ and $R T_{i j}$ values derived for each node as per Eqns. (10) and (11).

$$
\begin{gathered}
R P_{i j} \sim \operatorname{rnd}\left[P P_{i j .} P_{l}, 0.85 . P P_{i j .} P_{l}\right] \\
R T_{i j} \sim \operatorname{rnd}\left[P P T_{i j .} L T_{l .} f / V_{l}, 0.85 . P P T_{i j} . L T_{l} . f / V_{l}\right]
\end{gathered}
$$

The two reserved values are randomly selected from those falling within the specified upper and lower threshold values. The upper threshold of $R P_{i j}$ is calculated taking $P_{l}$ for each product from the corresponding consumer demand region and $P P_{i j}$ for each node. The lower threshold value is $85 \%$ of the upper threshold value. Similarly, $R T_{i j}$ is also a random value within the 
upper and lower threshold values, which are calculated based on the overall lead-time $\left(L T_{l}\right)$ as per the given product-market profile and the percentage time $\left(P P T_{i j}\right)$ allocated for each node. Here, $f$ is the dispatching frequency. The initial population for the GA is then subjected to the genetic operators, mutation and crossover, until the termination criteria are met.

Once the AU has generated a set of reserved prices and times representing each node to be used in the initial reverse-auctioning round, it starts the reverse-auctioning process by calling invitations. The invitations are sent to the physical agents through SESs. Reserved values for subsequent iterations are determined by lowering the initial set of reserved values by a certain percentage, and the reverse-auctioning continues until the termination criteria are met (i.e. on completion of a pre-defined number of iterations or when there are no more agents to bid).

The SES corresponding to each physical agent shortlists physical agents (i.e. $R_{i j k} \mathrm{~s}$ ) by comparing reserved values with the bids presented by physical agents in each iteration, as per Eqns. (12) and (13). Here, $z_{i j k}$ is a decision variable which has value 1 when the physical agent $R_{i j k}$ is shortlisted to fulfil a given product-market profile; otherwise it is 0 .

$$
\begin{aligned}
B P_{i j k .} z_{i j k} & \leq R P_{i j} \\
B T_{i j k .} z_{i j k} & \leq R T_{i j}
\end{aligned}
$$

At the end of the reverse-auctioning process, the OPT receives the final list of $R_{i j k} \mathrm{~S}$ representing shortlisted bids from the SES. Given this list of $R_{i j k} \mathrm{~s}$, the $\operatorname{cost}\left(T C_{i j k \rightarrow i^{\prime} j^{\prime} k^{\prime}}\right)$ and time $\left(T T_{i j k \rightarrow i^{\prime} j^{\prime} k^{\prime}}\right)$ representing the transportation function is obtained from the TA.

The TA generates transportation costs and times corresponding to a given product-market profile, which are calculated as per Eqns. (14) and (15) for a given SNC on the request of the OPT. Transportation cost is proportionate to the distance between $R_{i j k} \mathrm{~s}$. The distance between two selected physical agents $\left(R_{i j k}\right.$ and $\left.R_{i^{\prime} j^{\prime} k^{\prime}}\right)$ at two consecutive stages $i$ and $i^{\prime}(=i+1)$ is indicated by $D_{i j k \rightarrow i^{\prime} j^{\prime} k^{\prime}}$ and unit distance transportation cost is taken as $\propto_{2}$ and speed is taken as $V_{s}$. Here, $x_{i j k \rightarrow i^{\prime}{ }^{\prime} k^{\prime}}$ is the decision variable which has value 1 when $R_{i j k}$ in stage $i$ and $R_{i{ }^{\prime}{ }^{\prime} k^{\prime}}$ in stage $i^{\prime}$ are 
selected to fulfil a given order; otherwise it is 0 . A database is maintained by the TA, including a distance matrix, $\alpha_{2}$ and $V_{s}$.

$$
\begin{aligned}
T C_{i j k \rightarrow i^{\prime} j^{\prime} k^{\prime}} & =x_{i j k \rightarrow i^{\prime} j^{\prime} k^{\prime}} \cdot D_{i j k \rightarrow i^{\prime} j^{\prime} k^{\prime}} \cdot \alpha_{2} \cdot f \\
T T_{i j k \rightarrow i^{\prime} j^{\prime} k^{\prime}} & =\left(D_{i j k \rightarrow i^{\prime} j^{\prime} k^{\prime}} \cdot x_{i j k \rightarrow i^{\prime} j^{\prime} k^{\prime}}\right) / V_{S}
\end{aligned}
$$

The OPT then generates alternative optimal SNCs considering total SN costs (TSNC) and the overall lead-time (LT) satisfying the product-market profile of a given region.

The TSNC is the sum of the costs of individual operations (e.g. processing, assembly, storage and handling) at each $R_{i j k}$ and transportation costs between relevant $\mathrm{SN}$ stages. The overall LT of the SN is the sum of the: operations time of the selected $R_{i j k}$ in the final operational node $(j)$; transportation time between SN stages (i.e. $i$ and $i^{\prime}$ ); and the maximum delivery LT time of all connected nodes from the previous stage $i$ ', (= $i-1)$. Eqns. (16) and (17) represent the objectives of the OPT, which are to minimize TSNC and LT. The OPT achieves the above SNC objectives subject to the constraint expressed in Eqn. (18), which represents the selection of only one physical agent at each node to generate the SNC satisfying a given product-market

Minimize TSNC

$$
\begin{aligned}
= & \sum_{S_{i} \in S} \sum_{N_{i j} \in S_{i}} \sum_{R_{i j k} \in N_{i j}} y_{i j k} \cdot B P_{i j k} \cdot \delta_{i j} \cdot V_{l}+ \\
& \sum_{S_{i, i} \in S} \sum_{N_{i j} \in S_{i}} \sum_{N_{i^{\prime} j^{\prime}} \in S_{i}, R_{i^{\prime} j^{\prime} k^{\prime}} \in N_{i^{\prime} j^{\prime}}} x_{i j k \rightarrow i^{\prime} j^{\prime} k^{\prime}} \cdot D_{i j k \rightarrow i^{\prime} j^{\prime} k^{\prime}} \cdot \propto_{2} \cdot f
\end{aligned}
$$

$$
\begin{gathered}
=\sum_{R_{i j k} \in N_{i j}}^{\text {Minimize LT }} y_{i j k} B T_{i j k} \cdot \delta_{i j} \cdot V_{l} / f+\sum_{\substack{R_{i j k} \in N_{i j} \\
R_{i^{\prime} j^{\prime} k^{\prime}} \in N_{i^{\prime} j^{\prime}}}} x_{i j k \rightarrow i^{\prime} j^{\prime} k^{\prime}} \cdot D_{i j k \rightarrow i^{\prime} j^{\prime} k^{\prime}} / V_{S} \\
\quad+\max _{N_{i^{\prime \prime} j^{\prime \prime}} \in S_{i^{\prime \prime}}} L T_{i^{\prime \prime}}
\end{gathered}
$$

Subject to:

$$
\sum_{k \in K_{j}} y_{i j k}=1
$$


profile. Here, $y_{i j k}$ is a decision variable which has value 1 when the $R_{i j k}$ is selected to fulfil a given product-market profile; otherwise it is 0 .

In generating optimal alternative SNCs, NSGA-II starts with the initial population (i.e. parents), which is the set of SNCs having one entity option from each node, and follows the process outlined in Figure 5. The both objectives (TSNC and LT) are calculated to rank the population using the Pareto-fast non-dominated (PF-ND) sorting algorithm. Standard genetic operators (i.e. selection, crossover and mutation) are applied with a pre-determined criterion for ceasing the process of iterations. Elitism is achieved by combining the chosen attributes of parents and children, and are ranked with the use of PF-ND sorting. The next generation is selected from the highest-ranking population, which is then sent to the following generation. This process continues until the ceasing criterion is met. Finally, the solutions in the Pareto front are taken as the optimal alternative SNCs for a given product-market profile.

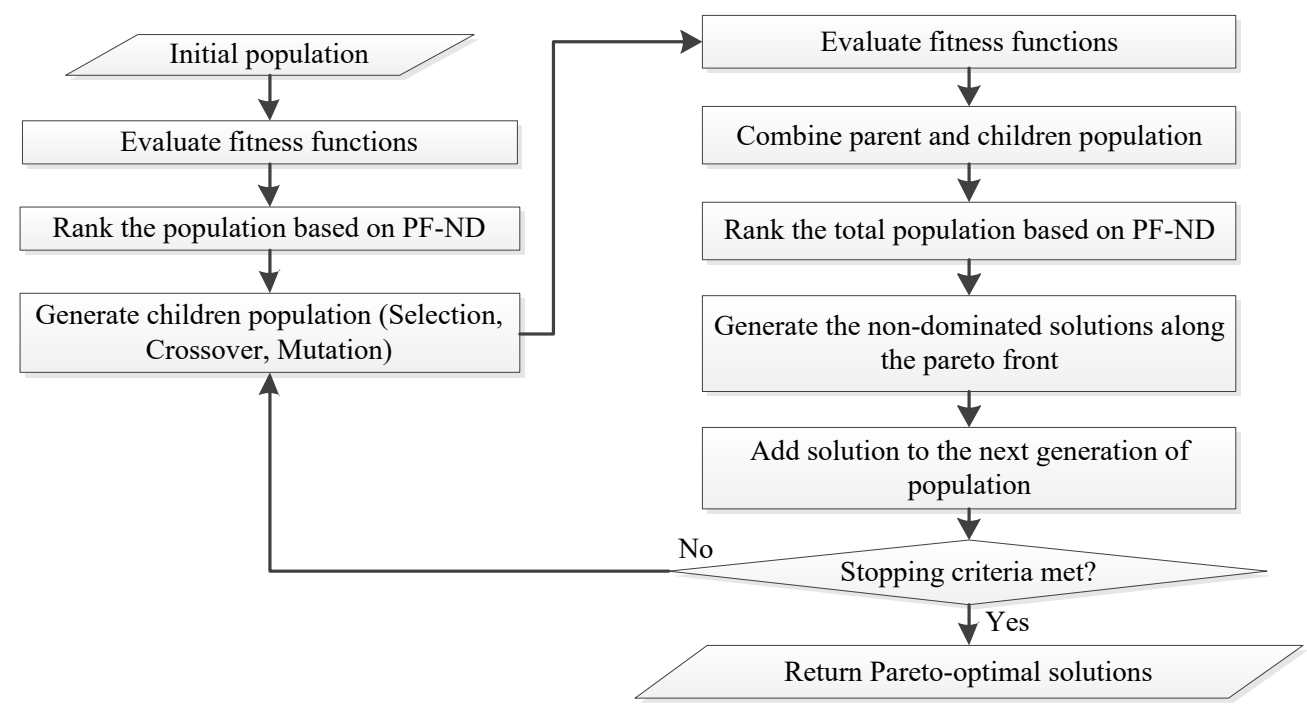

Figure 5: Conceptual flowchart of NSGA-II

The EA evaluates the alternative Pareto-optimal SNCs generated as above, based on additional criteria, and selects the most appropriate SNC to suit the given product-market profile considering other relevant conditions. After a particular SNC is selected, all physical 
agents are informed through relevant SESs to update their occupied production capacities (as in Eqn. 19), as well as the Q-table, using Eqn. 20, where $\mu_{i j k}^{3}>\mu_{i j k}^{1}>\mu_{i j k}^{2}$.

$$
\begin{gathered}
\lambda_{i j k}^{2}=\lambda_{i j k}^{2}+R C_{i j} /\left(1+\lambda_{i j k}^{1}\right) A C_{i j k} \\
Q_{m n}=Q_{m n}+\mu_{i j k}^{3}\left(\delta_{i j} \times V_{l} \times P_{m n}\right)
\end{gathered}
$$

\subsection{AGENT INTERACTIONS}

In the proposed multi-agent approach, agent interactions occur following CNP, which is a protocol widely applied in the context of distributed systems. Figure 6 shows the overall communication mechanism used in the MAOM, and Figure 7 shows agent connectivity. Figure 8 shows how information passes through relevant agents when performing the reverseauctioning process. Interactions are executed by the communication modules of each agent. Recognition: According to CNP, first, the necessity for breaking and sharing the main task into sub-tasks is recognised. In this study, catering to product-market profiles is the main task received by the OPT, which is then decomposed and allocated, via auxiliary agents, among the relevant supply nodes, based on the BOM.

Reverse-auctioning and bidding: Reverse-auctioning and bidding occur between the AU and physical agents through SESs. First, based on the BOM, the OP sends information regarding the supply nodes participating in the bidding process to the AU to generate a set of $R P_{i j} \mathrm{~s}$ and $R T_{i j \mathrm{~s}}$ for each $\mathrm{SN}$ node. As the reverse-auctioning begins, the SES sends invitations to relevant physical agents inviting bids for a given product-market profile. Upon receiving invitations, each physical agent follows its own strategies and procedures in preparing bids. These bids are then communicated to the SES to be compared against reserved values. This reverse-auctioning process continues through a series of bidding rounds (iterations) until the termination criteria are met. Finally, shortlisted bids are sent to the OPT so as to generate Pareto-optimal SNCs. Awarding: The OPT uses NSGA-II to find the Pareto-optimal SNCs considering the multiple bids received at each node. These SNCs are then sent to the EA, who determines the most 


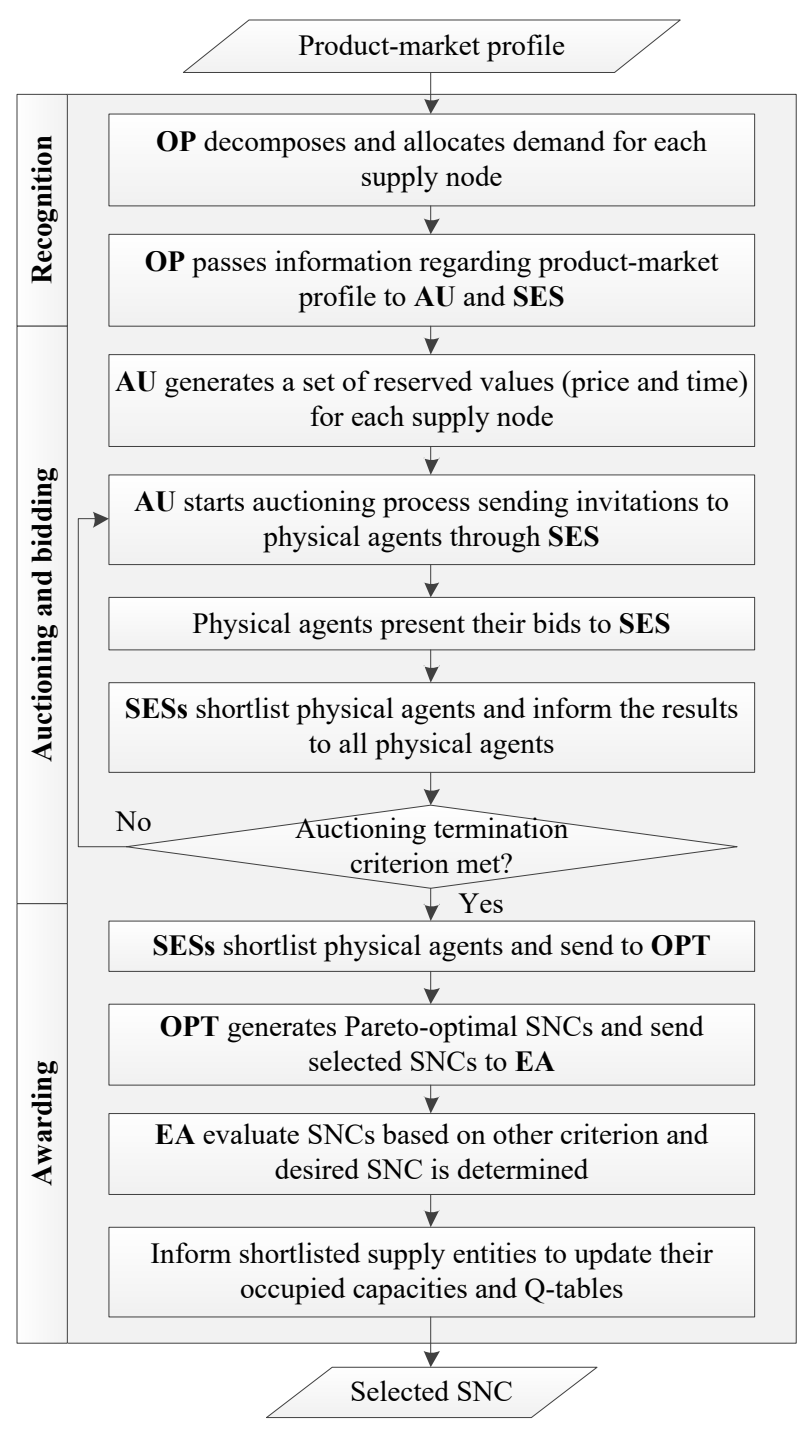

Figure 6: The process of CNP

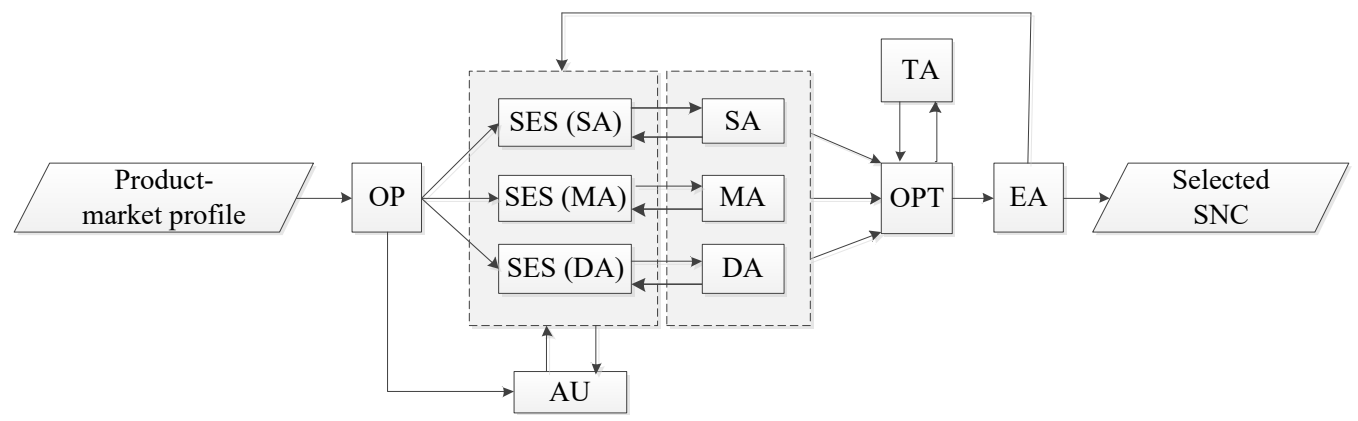

Figure 7: Agent connectivity in the overall system

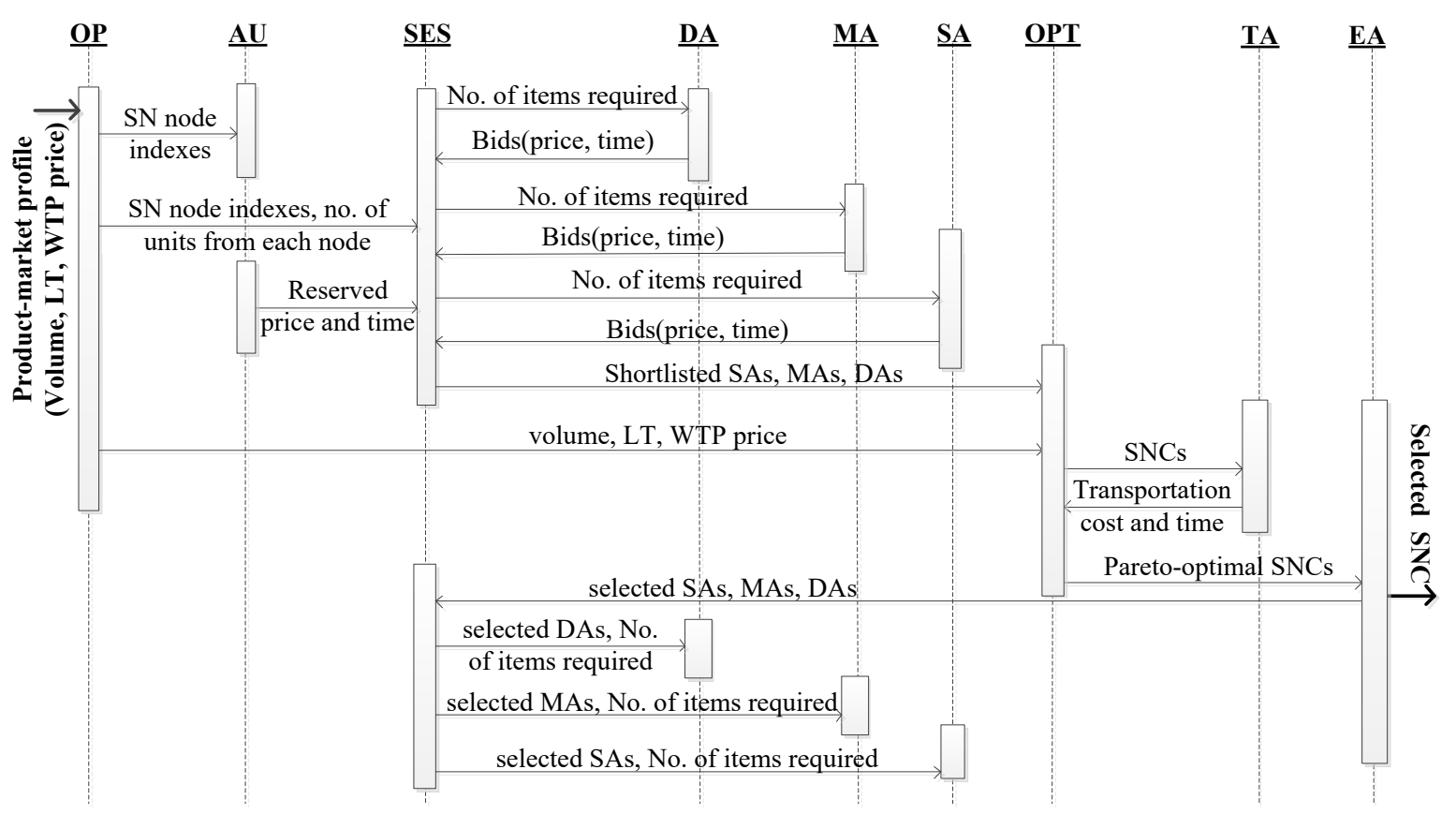

Figure 8: Information passing between agents (agent interactions) 
suitable SNC considering other possible criteria such as carbon emission and the compatibility between SN entities.

Once the desired SNC is determined, the outcome is passed to relevant physical agents so as to update their occupied production capacities and the Q-table.

\section{CASE STUDY ANALYSIS}

In this study, we adapted the dataset of a refrigerator production network, which was initially used by [55], and later modified by [56-57] by including additional parameters associated with the logistics networks to optimize lifecycle costs. To help demonstrate the efficacy of the proposed model, several new parameters are also added, considering the specific SNC problem considered in this paper. Further details about these parameters are presented below.

There are five stages in the refrigerator $\mathrm{SN}$; two supply stages (i.e. raw material and components), the final assembly stage and a distribution stage before finished products reaching the end-users via respective consumer regions (virtual retail outlets). There are 25 different components manufactured using four different types of raw material, namely, Iron, Plastic, Aluminium and Copper. Based on the type of raw material used and the nature of the manufacturing process involved, we categorize 25 components into five groups, aimed at reducing the complexity of the SN. The final assembled products are sent to distribution centres through which retailers at each consumer region receive goods. Accordingly, there are multiple nodes (i.e. $\mathbf{N}_{i j}$ ) in each stage, with multiple entity options (i.e. $R_{i j k}$ ) capable of performing the required value-adding functions at each node. The connectivity between nodes is shown in Figure 9. We consider seven consumer regions $(l=1-7)$ in Europe with distinct productmarket profiles attributed by $V_{l}, L T_{l}$ and $P_{l}$, which are derived using the four base parameters introduced in Section 3.1. Constructed variations in the attributes of the product-market profile as represented by their mean and standard deviation are given in Table 2 .

The behaviour of selected agents in making SNC decisions are illustrated in figures 10-12. 


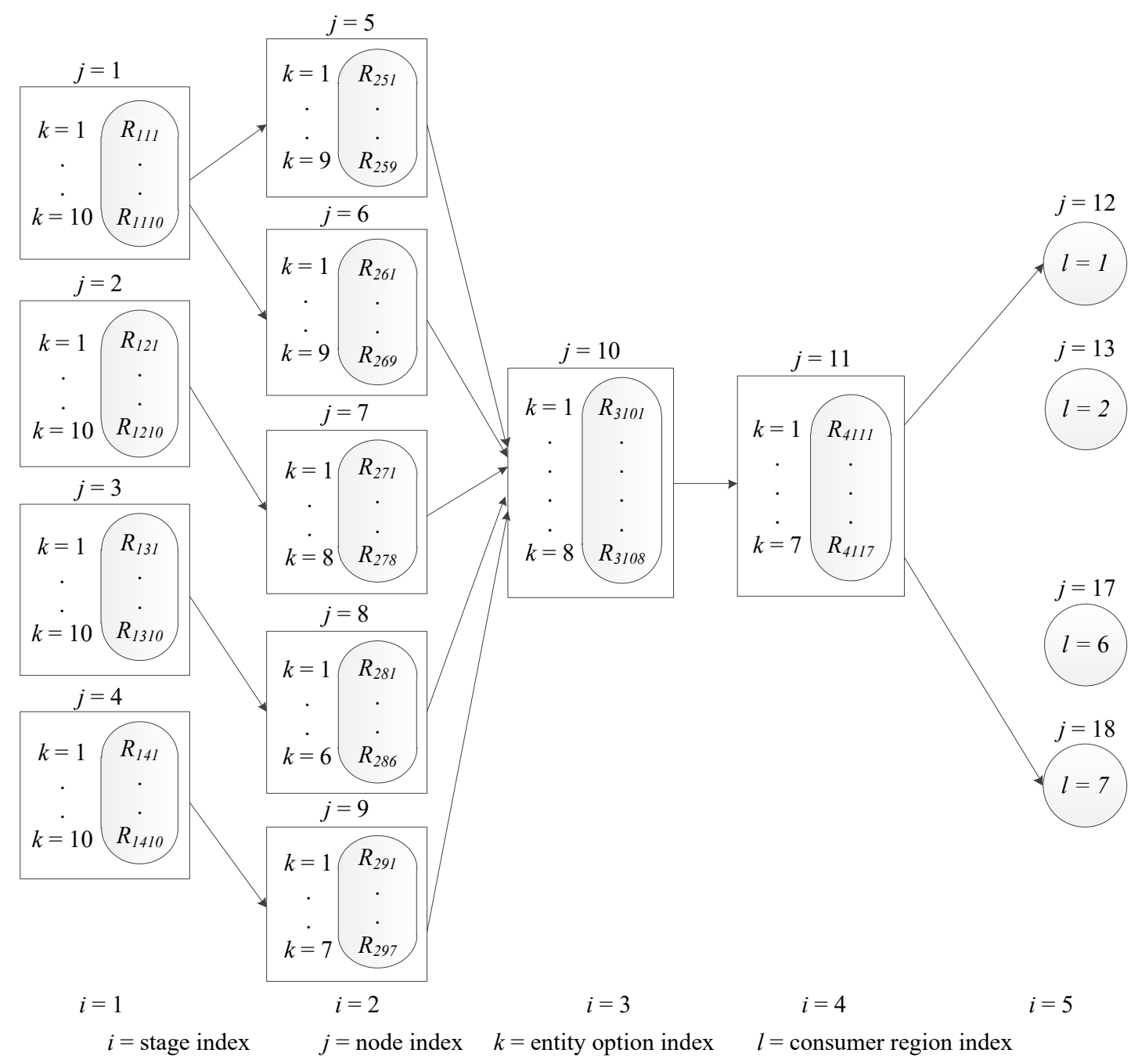

Figure 9: Illustration of the connectivity between nodes of the refrigerator production network

Table 2: Product-market profile of each consumer region

\begin{tabular}{|c|c|c|c|}
\hline$l$ & $\begin{array}{c}V_{l} \text { (units) } \\
(\text { mean,std) }\end{array}$ & $\begin{array}{c}L T_{l} \text { (days) } \\
\text { (mean,std) }\end{array}$ & $\begin{array}{c}P_{l} \text { (dollars) } \\
\text { (mean,std) }\end{array}$ \\
\hline 1 & $(15000,2500)$ & $(80,10)$ & $(1200,75)$ \\
\hline 2 & $(30000,4000)$ & $(150,15)$ & $(1300,45)$ \\
\hline 3 & $(35000,2000)$ & $(160,10)$ & $(1200,50)$ \\
\hline 4 & $(12000,1000)$ & $(70,12)$ & $(1200,40)$ \\
\hline 5 & $(19000,500)$ & $(100,5)$ & $(1200,55)$ \\
\hline 6 & $(57000,3000)$ & $(250,20)$ & $(1300,35)$ \\
\hline 7 & $(30000,1000)$ & $(150,15)$ & $(1300,30)$ \\
\hline
\end{tabular}

Figure 10 presents the optimal reserved values generated by the AU for the product-market profiles (using mean values only with no variation) of consumer regions 1 and 3 . Figure 11 shows the bidding decisions of the physical agent with ID 146 for three different product- 
market profiles and respective consumer regions. In this case, the physical agent (ID 146) offers bids by lowering the unit bidding prices for regions 1 and 3, and the agent bid only once for region 7. Figure 12 shows the optimal SNCs generated by the OPT catering to the productmarket profiles (using mean and standard deviation) of regions 1 and 3. Through these results, we demonstrated the autonomous behaviour of agents in the context of the proposed SNC

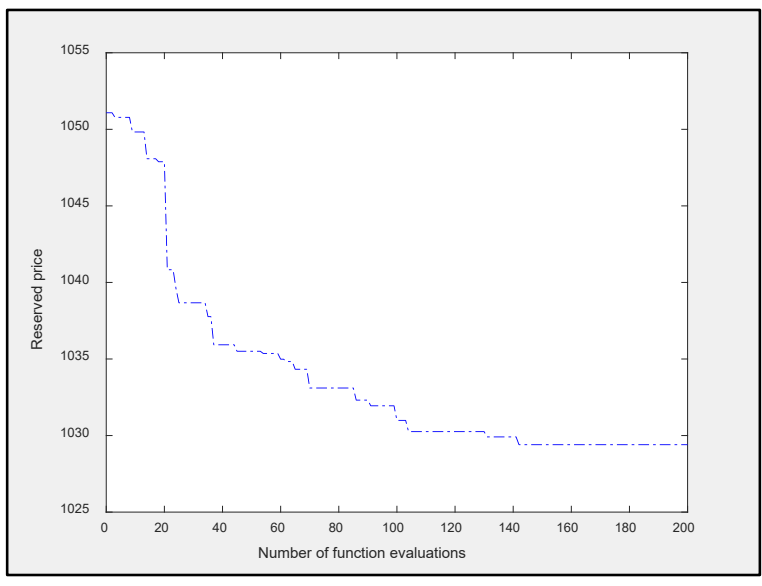

Region 1

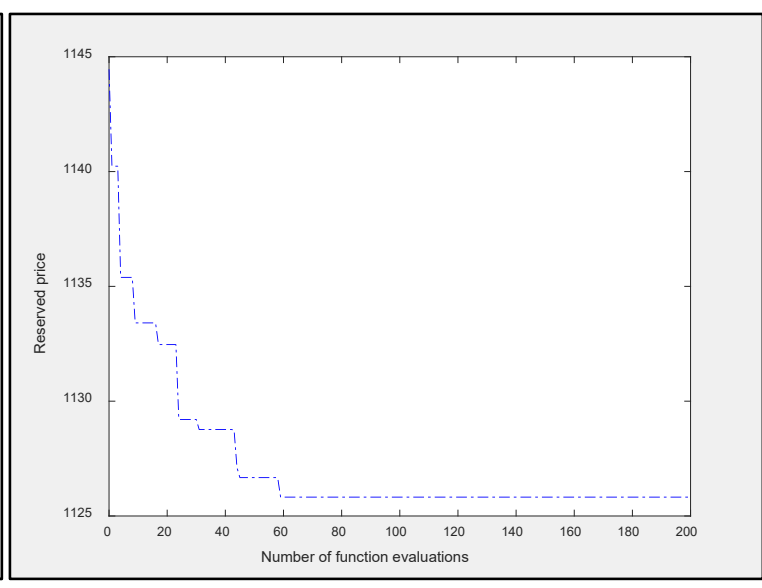

Region 3

Figure 10: Illustration of the iterative reserved prices generated by the AU (for consumer region 1and 3)

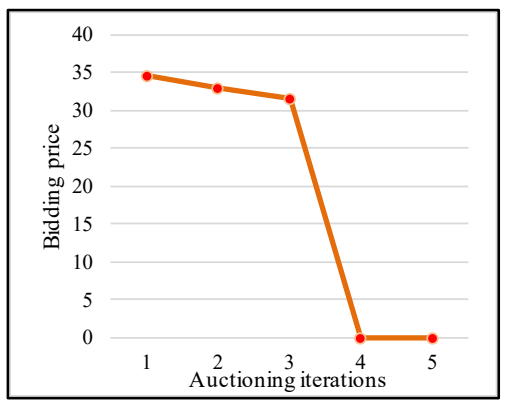

Region 1

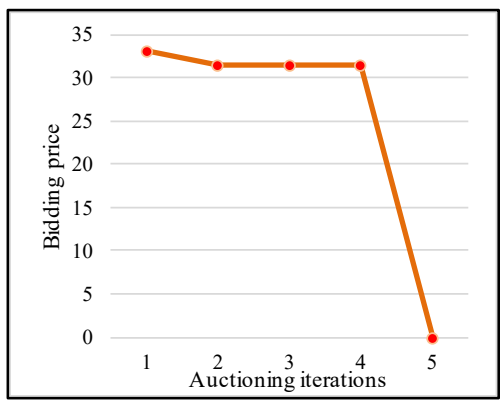

Region 3

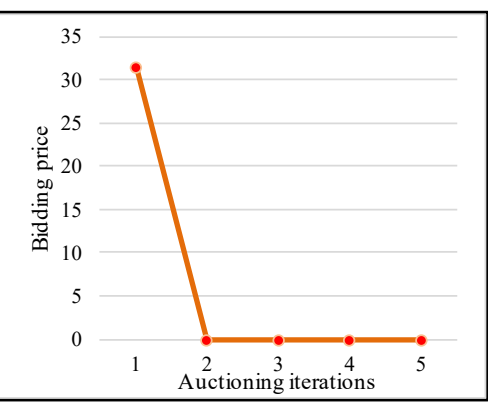

Region 7

Figure 11: Illustration of the unit-bidding price of Agent ID 146 (for consumer regions 1, 3, 7)

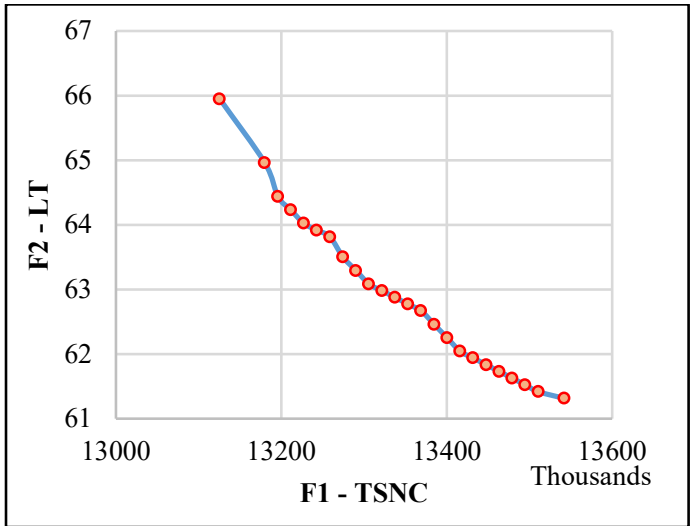

Region 1

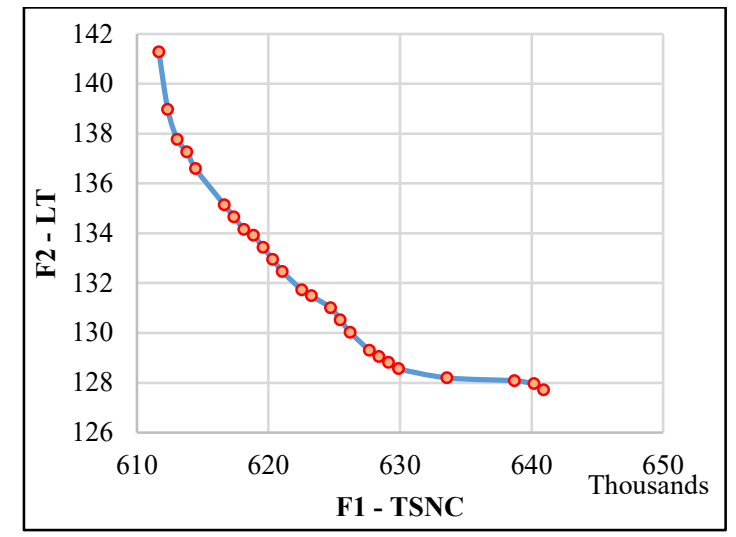

Region 3

Figure 12: Illustration of the Pareto-optimal solutions generated by the OPT (for consumer regions 1,3 ) 
problem, demonstrating their role and contribution to solving it.

We then conducted two experiments. In experiment 1, we considered a static product-market profile by employing only the mean value for each attribute of the respective product-market profile. In experiment 2, varied product-market profiles were used to create two scenarios with variations set using mean and standard deviation for all consumer regions. The proposed MAOM was tested to minimize both TSNC and LT.

Table 3: Solutions for static product-market profiles (Experiment 1)

\begin{tabular}{|c|c|c|c|c|c|}
\hline \multicolumn{2}{|c|}{ Experiment 1 setting } & \multicolumn{2}{c|}{ SNC solutions } & \multicolumn{2}{c|}{$\%$ Saving } \\
\hline$l$ & $\begin{array}{c}\text { Product-market profile } \\
\left(V_{l}, L T_{l,} P_{l}\right)\end{array}$ & TSNC range & LT range & Cost & Time \\
\hline 1 & $(15000,80,1200)$ & {$[866,877]$} & {$[61,63]$} & 27 & 22 \\
\hline 2 & $(30000,150,1300)$ & {$[874,903]$} & {$[110,115]$} & 32 & 25 \\
\hline 3 & $(35000,160,1200)$ & {$[874,907]$} & {$[128,141]$} & 26 & 16 \\
\hline 4 & $(12000,70,1200)$ & {$[878,900]$} & {$[45,52]$} & 26 & 31 \\
\hline 5 & $(19000,100,1200)$ & {$[886,910]$} & {$[73,78]$} & 25 & 24 \\
\hline 6 & $(57000,250,1300)$ & {$[883,911]$} & {$[207,233]$} & 31 & 12 \\
\hline 7 & $(30000,130,1300)$ & {$[886,915]$} & {$[111,124]$} & 31 & 22 \\
\hline
\end{tabular}

The results obtained in experiment 1 are presented in Table 3, which includes TSNC and LT ranges of the Pareto-optimal SNCs for each product-market profile. Potential gains (percentages) in both TSNC and LT are also calculated by comparing the average values of TSNC and LT ranges against corresponding product-market profile attributes $\left(P_{l}\right.$ and $\left.L T_{l}\right)$. The results from experiment 1 can help decision-makers to identify suitable SNCs in the presence of static product-market profiles. An appropriate SNC can then be selected from this set of Pareto-optimal SNCs considering any additional SN evaluation criteria, as desired.

Table 4 shows the results of experiment 2 with corresponding savings for all product-market profiles considered. The results shown in Table 3 indicate that the $\mathrm{SN}$ is capable of catering to product-market profiles (with mean values) of all 7 regions. However, the results in Table 4 indicate that certain product-market profiles cannot be fully catered to by the SNCs generated. For example, the lead-time of the product-market profile of consumer region 7 is infeasible. 
Table 4: Solutions for dynamic product-market profiles (Experiment 2)

\begin{tabular}{|c|c|c|c|c|c|}
\hline \multicolumn{2}{|c|}{ Experiment 2 setting } & \multicolumn{2}{c|}{ SNC solutions } & \multicolumn{2}{c|}{ \% Saving } \\
\hline \multirow{2}{*}{$l$} & $\begin{array}{c}\text { Product-market profile } \\
\left(V_{l,} L T_{l}, P_{l}\right)\end{array}$ & TSNC range & LT range & Cost & Time \\
\hline \multirow{2}{*}{1} & $(16000,85,1295)$ & {$[867,877]$} & {$[60,63]$} & 33 & 27 \\
\cline { 2 - 6 } & $(14500,83,1230)$ & {$[863,877]$} & {$[61,62]$} & 30 & 26 \\
\hline \multirow{2}{*}{2} & $(32000,160,1342)$ & {$[875,910]$} & {$[109,114]$} & 33 & 30 \\
\cline { 2 - 7 } & $(26600,147,1350)$ & {$[877,900]$} & {$[110,113]$} & 34 & 24 \\
\hline \multirow{2}{*}{3} & $(34200,165,1250)$ & {$[873,905]$} & {$[128,142]$} & 29 & 18 \\
\cline { 2 - 7 } & $(36600,153,1300)$ & {$[874,910]$} & {$[129,141]$} & 31 & 12 \\
\hline \multirow{2}{*}{4} & $(12300,65,1210)$ & {$[877,903]$} & {$[45,52]$} & 25 & 25 \\
\cline { 2 - 7 } & $(12700,72,1170)$ & {$[878,903]$} & {$[45,53]$} & 24 & 32 \\
\hline \multirow{2}{*}{5} & $(18700,103,1220)$ & {$[884,910]$} & {$[73,78]$} & 26 & 27 \\
\cline { 2 - 7 } & $(19100,110,1310)$ & {$[886,909]$} & {$[74,78]$} & 31 & 31 \\
\hline \multirow{2}{*}{6} & $(58200,243,1330)$ & {$[885,913]$} & {$[208,234]$} & 32 & 9 \\
\cline { 2 - 7 } & $(54100,255,1370)$ & {$[880,912]$} & {$[205,234]$} & 34 & 14 \\
\hline \multirow{2}{*}{7} & $(31500,120,1280)$ & {$[887,914]$} & {$[111,125]$} & 29 & -2 \\
\cline { 2 - 7 } & $(30100,115,1200)$ & {$[888,915]$} & {$[114,124]$} & 25 & -3 \\
\hline
\end{tabular}

The results obtained in this analysis can be used to find a strategically robust SNC, which can withstand the effects of volatile markets that are characterized by varied $V_{l}, L T_{l}$ and $P_{l}$ values or when faced with the disruptions discussed earlier. The proposed approach allows for the amendment of criteria used in evaluating SNCs to reflect changing strategic priorities, the adjustment of product-market profile attributes to reflect organisational and/or environmental conditions or undertake the reverse-auctioning process under re-negotiated terms of supply, in cases where the existing supply base is found to be unable to meet the expected criteria.

\section{CONCLUSION}

In this study, we demonstrated the application of a novel multi-agent modelling approach to support SNC decisions, addressing three key challenges reported in the literature. The first and most significant challenge is enhancing SN-level performance in a distributed decision-making environment, where individual SN entities aiming to satisfy their local goals (i.e. competitive 
priorities). The second challenge is achieving the above goals with minimal information sharing between SN entities, which reflects the real-world situation of organisations' reluctance to disclose commercially sensitive information. The third challenge is sustaining competitive SNs in a dynamic business environment, which is characterised by shifting consumer requirements, disruptions and other forms of uncertainty.

To this end, we modelled a multi-stage, multi-echelon SN consisting of a geographically dispersed set of SN entities catering to distinct product-market profiles. In modelling this SNC decision problem, we used two types of agents, physical and auxiliary, with each type having distinct characteristics and architectures, as well as playing specific roles in relation to making SNC decisions. The proposed MAOM incorporated a reverse-auctioning process to simulate the competing nature of SN entities with respect to achieving individual organisational-level goals but collectively contributing to enhancing SN-level performance, by means of setting reserve values generated through the application of GA. Then, with respect to generating Pareto-optimal SNCs capable of catering to distinct product-market profiles, we employed NSGA-II. Further evaluation of these SNCs against additional criteria using a rule-based approach allowed the selection of the most appropriate SNC to meet a broader set of conditions.

We tested the model on a refrigerator SN case study drawn from the literature to demonstrate its efficacy. Experimental results revealed that a number of strategic decisions can be supported by the proposed model, in particular, identifying and evaluating robust SNs to suit varied product-market profiles. Additionally, in identifying and evaluating these SNCs, we only used product-market profile data and SN entity-level bidding prices and and times, meaning the model can be run with minimal input data. We further contend that dealing with SNC decisions in the manner outlined in this paper can enhance SN capabilities in terms of their capacity to withstand anticipated disruptions, as well as developing contingencies in the case of having to recover from disruptions. Further opportunities exist for extending and expanding this research 
to investigate the merits or otherwise of alternative modelling approaches and algorithms such as ROs/Game Theory [e.g. 13], Dynamic Metaheuristics [e.g. 58] and improved Bayesian frameworks [e.g. 59-60] against those used in the proposed model. These alternative approaches and algorithms, which have already been tested in other related areas under comparable conditions could be used to mimic the competition and/or cooperation between SN entities, with the possibility for handling more complex SNs at different levels of abstraction.

In terms of the contribution of our work to knowledge, we claim that there is a distinct advantage in applying this type of decision support tools in relation to enhancing SNC decisionmaking. Compared to the existing decision support tools, the proposed approach effectively addresses the three key challenges referred to earlier, which we believe is a significant contribution to practice. Potentially, this model can be used to enhance SNC decisions by any SN entity, as well as other parties such as SC analysts or consultants. In terms of contribution to theory, the use of this model will certainly help better understand SN dynamics at a fundamental level and evaluate alternative scenarios for understanding the sensitivity of model outcomes to certain parameters that represent the features of real-world SNs. Given the limitations of the scope, including space, of this paper, we plan to report the results of our broader study, which includes sensitivity analysis, as well as further testing of our model with larger data sets, on more complex SNs and in other contexts, through future publications.

\section{APPENDIX I - Application of AHP to Calculate Aggregate Demand $\left(V_{l}\right)$}

The 5-step AHP process followed to estimate $V_{l}$ is outlined below.

Step 1- Identify the goal, criteria and alternatives: The goal of using AHP is to estimate the demand for each consumer region in the refrigerator SN. The four criteria $\left(C_{i}\right)$ used are per capita income, energy consumption, price level index and population density. The alternatives $\left(A_{j}\right)$ considered are seven consumer regions in Europe namely, Zaragoza, Milan, Munich, Hannover, Nuremberg, Paris, and Prague. 
Step 2 - Collect data to rate criteria and alternatives: The criteria identified in this study requires rating based on subjective opinion which needs to be converted into numerical values. The scale commonly used as proposed by Saaty is: 1-3-5-7-9 which stand for equally importance, mildly stronger, stronger, much stronger, and extremely stronger. With respect to consumer regions, ratings were derived using statistical data. Then the pairwise comparison matrix is constructed for both criteria and alternatives to indicate how much one criterion is important compared to another.

Step 3 - Calculate the weight of each criterion $\left(w_{i}\right)$ and the weight of consumer regions with respect to each criterion $\left(w_{i j}\right)$ : Both $w_{i}$ and $w_{i j}$ are calculated by using the normalized Eigen vector of the pairwise comparison matrix constructed for criteria and alternatives. The elements in the normalized Eigen vector are relevant weights of each criteria. Similarly, the ratio-scale weight between the pair of alternatives for each criterion is calculated using statistical data. For the given criterion $i$, compare $j$ number of alternatives and construct the square matrix and then find the normalized Eigen vector. The elements in the normalized Eigen vector are the relevant weights (i.e. $w_{i j}$ ) of each alternative.

Step 5 - Calculate the additive weight for each consumer region with respect to all criteria: The derived weights $\left(w_{i j}\right)$ of each consumer region for a given criteria is multiplied by the weight of the respective criteria $w_{i}$ and aggregate it to obtain the additive weight $\left(W_{j}\right)$. The additive weight of the alternative $j$ is $W_{j}=w_{l j} w_{1}+w_{2 j} w_{2}+\ldots+w_{m j} w_{m}$

The calculated $W_{j}$ for each consumer region is used in estimating the annual aggregate demand of the respective consumer region.

\section{REFERENCES}

[1] D. Mourtzis and M. Doukas, "Decentralized manufacturing systems review: challenges and outlook," in Robust Manufacturing Control, Springer, 2013, pp. 355-369. 
[2] B. Tjahjono, C. Esplugues, E. Ares and G. Pelaez, "What does industry 4.0 mean to supply chain?," Procedia Manufacturing, vol. 13, pp. 1175-1182, 2017.

[3] K. Govindan, M. Fattahi, and E. Keyvanshokooh, "Supply chain network design under uncertainty: A comprehensive review and future research directions," European Journal of Operational Research, vol. 263, no. 1, pp. 108-141, 2017.

[4] T. G. Schmitt, S. Kumar, K. E. Stecke, F. W. Glover, and M. A. Ehlen, "Mitigating disruptions in a multi-echelon supply chain using adaptive ordering," Omega, vol. 68, pp. 185-198, 2017.

[5] S. Rezapour, R. Z. Farahani, and M. Pourakbar, "Resilient supply chain network design under competition: a case study," European Journal of Operational Research, vol. 259, no. 3, pp. 1017-1035, 2017.

[6] K. Govindan and M. Fattahi, "Investigating risk and robustness measures for supply chain network design under demand uncertainty: A case study of glass supply chain," International Journal of Production Economics, vol. 183, pp. 680-699, 2017.

[7] E. Revilla and M. J. Saenz, "The impact of risk management on the frequency of supply chain disruptions: a configurational approach," International Journal of Operations \& Production Management, vol. 37, no. 5, pp. 557-576, 2017.

[8] M. Kamalahmadi and M. M. Parast, "A review of the literature on the principles of enterprise and supply chain resilience: Major findings and directions for future research," International Journal of Production Economics, vol. 171, pp. 116-133, 2016.

[9] S. Y. Ponomarov and M. C. Holcomb, "Understanding the concept of supply chain resilience," The international journal of logistics management, vol. 20, no. 1, pp. 124-143, 2009. 
[10] S. E. Birkie, P. Trucco, and P. Fernandez Campos, "Effectiveness of resilience capabilities in mitigating disruptions: leveraging on supply chain structural complexity," Supply Chain Management: An International Journal, vol. 22, no. 6, pp. 506-521, 2017.

[11]A. Hasani and A. Khosrojerdi, "Robust global supply chain network design under disruption and uncertainty considering resilience strategies: A parallel memetic algorithm for a real-life case study," Transportation Research Part E: Logistics and Transportation Review, vol. 87, pp. 20-52, 2016.

[12] Braziotis, M. Bourlakis, H. Rogers, and J. Tannock, "Supply chains and supply networks: distinctions and overlaps," Supply Chain Management: An International Journal, vol. 18, no. 6 , pp. 644-652, 2013.

[13]L. Trigeorgis and A.E. Tsekrekos, "Real options in operations research: A review," European Journal of Operational Research, vol.270, no.1, pp.1-24, 2018.

[14] R. H. Ballou, "Unresolved issues in supply chain network design," Information Systems Frontiers, vol. 3, no. 4, pp. 417-426, 2001.

[15] S. A. Melnyk, R. Narasimhan and H. A. DeCampos, "Supply chain design: issues, challenges, frameworks and solutions," International Journal of Production Research, vol.52, no.7, pp.1887-1896, 2014.

[16] S. Dharmapriya, S. Kiridena and N. Shukla, “ Modelling sustainable supply networks with adaptive agents," in International Conference on Production and Operations Management Society (POMS), 2018, pp. 1-8.

[17] S. Piramuthu, "Machine learning for dynamic multi-product supply chain formation," Expert Systems with Applications, vol. 29, no. 4, pp. 985-990, 2005.

[18] T. H. Truong and F. Azadivar*, "Optimal design methodologies for configuration of supply chains," International Journal of Production Research, vol. 43, no. 11, pp. 2217 2236, 2005. 
[19] T. P. Harrison, "Global supply chain design," Information Systems Frontiers, vol. 3, no. 4, pp. 413-416, 2001.

[20] M. J. Meixell and V. B. Gargeya, "Global supply chain design: A literature review and critique," Transportation Research Part E: Logistics and Transportation Review, vol. 41, no. 6 , pp. 531-550, 2005.

[21]N. Azad, H. Davoudpour, G. K. Saharidis, and M. Shiripour, "A new model to mitigating random disruption risks of facility and transportation in supply chain network design," The International Journal of Advanced Manufacturing Technology, vol. 70, no. 9-12, pp. 1757 1774, 2014.

[22] C. Chandra and J. Grabis, "Reconfigurable supply chains: an integrated framework," in Supply Chain Configuration, Springer, 2016, pp. 69-86.

[23]X. Yao and R. Askin, "Review of supply chain configuration and design decision-making for new product," International Journal of Production Research, vol. 57, no.7, pp.22262246, 2019.

[24]D. J. Garcia and F. You, "Supply chain design and optimization: Challenges and opportunities," Computers \& Chemical Engineering, vol. 81, pp.153-170, 2015.

[25]L. A. Moncayo-Martínez and D. Z. Zhang, "Multi-objective ant colony optimisation: a meta-heuristic approach to supply chain design," International Journal of Production Economics, vol. 131, no. 1, pp. 407-420, 2011.

[26]B. Yuce, E. Mastrocinque, A. Lambiase, M. S. Packianather, and D. T. Pham, "A multiobjective supply chain optimisation using enhanced Bees Algorithm with adaptive neighbourhood search and site abandonment strategy," Swarm and Evolutionary Computation, vol. 18, pp. 71-82, 2014. 
[27] M. Wang, H. Wang, D. Vogel, K. Kumar, and D. K. Chiu, "Agent-based negotiation and decision-making for dynamic supply chain formation," Engineering Applications of Artificial Intelligence, vol. 22, no. 7, pp. 1046-1055, 2009.

[28]J. R. Jiao, X. You, and A. Kumar, "An agent-based framework for collaborative negotiation in the global manufacturing supply chain network," Robotics and ComputerIntegrated Manufacturing, vol. 22, no. 3, pp. 239-255, 2006.

[29]P. Lou, Y.-P. Chen, and W. Ai, "Study on multi-agent-based agile supply chain management," The international journal of advanced manufacturing technology, vol. 23, no. 3-4, pp. 197-203, 2004.

[30] O. Akanle and D. Zhang, "Agent-based model for optimising supply-chain configurations," International Journal of Production Economics, vol. 115, no. 2, pp. $444-$ 460, 2008.

[31]N. Shukla and S. Kiridena, "A fuzzy rough sets-based multi-agent analytics framework for dynamic supply chain configuration," International Journal of Production Research, vol. 54, no. 23, pp. 6984-6996, 2016.

[32]F. Ameri and C. McArthur, "A multi-agent system for autonomous supply chain configuration," The International Journal of Advanced Manufacturing Technology, vol. 66, no. 5-8, pp. 1097-1112, 2013.

[33]L. Greco, L. L. Presti, A. Augello, G. L. Re, M. La Cascia, and S. Gaglio, "A decisional multi-agent framework for automatic supply chain arrangement," in New Challenges in Distributed Information Filtering and Retrieval, Springer, 2013, pp. 215-232.

[34] W. Klibi, A. Martel, and A. Guitouni, "The design of robust value-creating supply chain networks: A critical review," European Journal of Operational Research, vol. 203, no. 2 , pp. 283-293, 2010. 
[35] World Economic and Financial Surveys, International Monetary Fund, July 2017. [Online].

Available:https://www.imf.org/external/pubs/ft/weo/2018/01/weodata/index.aspx

[36] Comparative price levels of consumer goods and services, Eurostat statistics explained. [Online].Available:https://ec.europa.eu/eurostat/statisticsexplained/index.php/Comparati ve_price_levels_of_consumer_goods_and_services\#Price_levels_for_energy.2C_furnitur e.2C_household_appliances_and_consumer_electronics

[37]Electric power consumption (kWh per capita), The World Bank Group. [Online]. Available: https://data.worldbank.org/indicator/EG.USE.ELEC.KH.PC

[38]European Countries by population (2018), Worldometers. [Online]. Available: http://www.worldometers.info/population/countries-in-europe-by-population/

[39] J. M. Swaminathan, S. F. Smith, and N. M. Sadeh, "Modeling supply chain dynamics: A multiagent approach," Decision sciences, vol. 29, no. 3, pp. 607-632, 1998.

[40] M. Barbati, G. Bruno, and A. Genovese, "Applications of agent-based models for optimization problems: A literature review," Expert Systems with Applications, vol. 39, no. 5 , pp. $6020-6028,2012$.

[41] S. Russell, P. Norvig, "Artificial Intelligence: A modern approach," Malaysia: Pearson Education Limited, 2016.

[42] S.A. Mostafa, M.S. Ahmad, A. Mustapha and M.A. Mohammed, "A concise overview of software agent research, modeling, and development," Software Engineering, vol. 5, no. 1, pp. 8-25, 2017.

[43]P. Marks, T. Müller, D. Vögeli, T. Jung, N. Jazdi and M. Weyrich, "Agent Design Patterns for Assistance Systems in Various Domains-a Survey," 2018 IEEE 14th International Conference on Automation Science and Engineering (CASE), pp. 168-73, 2018. 
[44] J.-H. Lee and C.-O. Kim, "Multi-agent systems applications in manufacturing systems and supply chain management: a review paper," International Journal of Production Research, vol. 46, no. 1, pp. 233-265, 2008.

[45]D. Juneja, A. Singh, R. Singh and S. Mukherjee, "A thorough insight into theoretical and practical developments in multiagent systems," International Journal of Ambient Computing and Intelligence (IJACI), vol. 8, no. 1, pp. 23-49, 2017.

[46] C.M. Macal and M.J. North, "Tutorial on agent-based modelling and simulation", Journal of Simulation, vol. 4, no. 3, pp. 151-62, 2010.

[47]M. Van Otterlo, "Markov Decision Processes: Concepts and Algorithms," Course on Learning and Reasoning, 2009.

[48] S. Serdarasan, "A review of supply chain complexity drivers," Computers \& Industrial Engineering, vol. 66, no. 3, pp. 533-540, 2013.

[49] M. Wooldridge and N. R. Jennings, "Intelligent agents: Theory and practice," The knowledge engineering review, vol. 10, no. 2, pp. 115-152, 1995.

[50] K. O. Chin, K. S. Gan, R. Alfred, P. Anthony, and D. Lukose, "Agent Architecture: An Overviews," Transactions on science and technology, vol. 1, no. 1, pp. 18-35, 2014.

[51]P. Maes, "The agent network architecture (ANA)," Acm sigart bulletin, vol. 2, no. 4, pp. $115-120,1991$.

[52] M. Caridi and S. Cavalieri, "Multi-agent systems in production planning and control: an overview," Production Planning \& Control, vol. 15, no. 2, pp. 106-118, 2004.

[53] M. L. Puterman, "Markov decision processes: discrete stochastic dynamic programming," John Wiley \& Sons, 2014.

[54] R.S. Sutton and A.G. Barto, "Reinforcement learning: An introduction,” MIT press, 1998.

[55] Y. Umeda, A. Nonomura, and T. Tomiyama, "Study on life-cycle design for the post mass production paradigm," AI EDAM, vol. 14, no. 2, pp. 149-161, 2000. 
[56]H. R. Krikke, J. M. Bloemhof-Ruwaard, and L. N. Van Wassenhove, "Dataset of the Refrigerator Case: design of closed loop supply chains," ERIM Report Series Reference No. ERS-2001-46-LIS, 2001.

[57] M. Fleischmann, P. Beullens, J. M. Bloemhof-Ruwaard, and L. N. Van Wassenhove, "The impact of product recovery on logistics network design," Production and operations management, vol. 10, no. 2, pp. 156-173, 2001.

[58] S. Yang, Y. Jiang, and T. T. Nguyen, "Metaheuristics for dynamic combinatorial optimization problems," IMA Journal of Management Mathematics, vol. 24, no. 4, pp. 451-480, 2012.

[59] M. Imani, S. F. Ghoreishi, D. Allaire, and U. M. Braga-Neto, "MFBO-SSM: Multi-fidelity Bayesian optimization for fast inference in state-space models," in Proceedings of the AAAI Conference on Artificial Intelligence, 2019, vol. 33, pp. 7858-7865.

[60] M. Imani, S. F. Ghoreishi, and U. M. Braga-Neto, "Bayesian control of large MDPs with unknown dynamics in data-poor environments," in Advances in neural information processing systems, 2018, pp. 8146-8156. 